\title{
Differences in Lung and Lobe Volumes between Supine and Standing Positions Scanned with Conventional and Newly Developed 320-Detector-Row Upright CT: Intra-Individual Comparison
}

\author{
Yoshitake Yamada ${ }^{a}$ Minoru Yamada ${ }^{a}$ Yoichi Yokoyama ${ }^{a}$ Akiko Tanabe $^{b}$ \\ Shiho Matsuoka ${ }^{b}$ Yuki Niijimac Keiichi Narita ${ }^{c}$ Takehiro Nakahara ${ }^{a}$ \\ Mitsuru Muratad Koichi Fukunaga ${ }^{\mathrm{e}}$ Shotaro Chubachi ${ }^{\mathrm{e}}$ Masahiro Jinzaki ${ }^{\mathrm{a}}$ \\ ${ }^{a}$ Department of Radiology, Keio University School of Medicine, Tokyo, Japan; b Department of Clinical Laboratory, \\ Keio University Hospital, Tokyo, Japan; ' Office of Radiation Technology, Keio University Hospital, Tokyo, Japan; \\ ${ }^{d}$ Department of Laboratory Medicine, Keio University School of Medicine, Tokyo, Japan; e Division of Pulmonary \\ Medicine, Department of Medicine, Keio University School of Medicine, Tokyo, Japan
}

\section{Keywords}

Lung volume measurements · Multidetector computed tomography $\cdot$ Posture $\cdot$ Standing position $\cdot$ Thorax

\footnotetext{
Abstract

Background: No clinical studies to date have compared unilateral lung or lobe volumes between the supine and standing positions. Objectives: To compare lung/lobe volumes on computed tomography (CT) between these two positions and evaluate the correlation between the total lung volume and total lung capacity (TLC) on pulmonary function tests (PFTs). Methods: Thirty-two asymptomatic volunteers underwent both conventional CT (supine position) and upright $\mathrm{CT}$ (standing position), during deep inspiration breath-hold, and PFTs on the same day. We measured lung/lobe volumes on CT in each position. Paired $t$ tests were used for statistical analysis. Results: The volumes of the total lung (10.9\% in-
}

crease), right lung (10.3\% increase), right upper lobe $(8.6 \%$ increase), right lower lobe (14.6\% increase), left lung $(11.6 \%$ increase), left upper lobe (7.1\% increase), and left lower lobe (16.0\% increase) were significantly greater in the standing position than in the supine position (all $p<0.0001$ ). The right middle lobe volume was similar between the two positions $(p=0.16)$. Intraclass correlation coefficients for agreement between total lung volumes on CT in the supine/standing positions and the TLC on PFT were 0.891/0.938, respectively. Conclusions: While the volumes of the bilateral upper and lower lobes and bilateral lungs were significantly greater in the standing than in the supine position, with lower lobes showing larger changes, the right middle lobe volume did not change significantly between positions. The total lung volume on upright $C T$ in the standing position was more similar to TLC on PFT than that in the supine position.

C 2020 The Author(s)

Published by S. Karger AG, Basel

$\begin{array}{ll}\text { karger@karger.com } & \text { (c) 2020 The Author(s) } \\ \text { Pww.karger.com/res } & \text { Published by S. Karger AG, Basel } \\ \text { This article is licensed under the Creative Commons Attribution- } & \text { NonCommercial-NoDerivatives 4.0 International License (CC BY- } \\ \text { NC-ND) (http://www.karger.com/Services/OpenAccessLicense). } \\ \text { Usage and distribution for commercial purposes as well as any dis- } \\ \text { tribution of modified material requires written permission. }\end{array}$

Yoshitake Yamada

Department of Radiology

Keio University School of Medicine

35 Shinanomachi, Shinjuku-ku, Tokyo 160-8582 (Japan)

yamada@rad.med.keio.ac.jp 


\section{Introduction}

Although humans spend most of their day in an upright position, the basic anatomy of the human lung in the upright position is not yet well understood. Chest radiography is the most commonly used technique for the diagnosis and follow-up of pulmonary diseases and can be acquired in a standing position; however, chest radiography provides a two-dimensional image and cannot accurately depict the volume of organs, such as the lungs or heart. While cone-beam computed tomography (CT) can provide cross-sectional images in a standing position, $>10 \mathrm{~s}$ are needed to acquire one axial conebeam CT image because of its slow rotation time $[1,2]$. This is quite a long period of time for a subject to stand still without support and could result in an inaccurate quantitative analysis of images because of motion artifacts $[1,2]$. Furthermore, the field of view of cone-beam CT for the upright position is limited $[1,2]$ and is insufficient to cover the whole lungs at present. Moreover, cone-beam CT has limited low-contrast resolution due to various physical and technical factors, which limits soft tissue evaluation $[3,4]$, and suffers from poor image quality and inaccurate CT numbers compared with multidetector-row CT $[5,6]$.

To overcome the limitations of these imaging modalities for evaluation of human anatomy in the upright position and to clarify the effects of gravity on the entire human body, a 320-detector-row upright CT scanner has recently been developed. This scanner allows up-anddown movements of a transverse 320-detector-row gantry (isotropic $0.5-\mathrm{mm}$ detector size), with a maximum gantry rotation speed of $0.275 \mathrm{~s}$, a $780-\mathrm{mm}$ bore, and a field of view of $500 \mathrm{~mm}$ [7]. This upright CT scanner allows for the acquisition of isotropic volume data of the whole chest within approximately $5 \mathrm{~s}$.

It has been reported that the total lung capacity (TLC) measured by pulmonary function tests (PFTs) using spirometry in the standing position is higher than that obtained in the supine position $[8,9]$; however, neither the unilateral lung volume nor the volume of each lung lobe can be measured by PFT. On the other hand, CT volumetry can provide the volumes of the unilateral lung and each lobe of the lung [10-13] and has been reported to enable more accurate predictions of postoperative residual pulmonary function in patients with lung cancer than the segment-counting method, which is based solely on the number of remaining pulmonary segments [10-12]. However, CT volumetry with conventional CT is performed in the supine position and does not reflect daily life conditions, whereas PFT is usually performed in the sitting (upright) position, which is a daily-life posture.

To the best of our knowledge, no clinical studies to date have accurately evaluated the volumes of each lung or of each lobe of the lung in the standing position. We hypothesized that the volume change rates between supine and standing positions would be different for each lobe of the lung, and that the proportional volume of each lobe relative to the total lung volume would be different between supine and standing positions, because the direction of gravity relative to the chest differs between these positions.

The purpose of this study was thus to compare lung and lung lobe volumes on CT between the supine and standing positions and to evaluate the correlation between the total lung volume on CT and TLC on PFT.

\section{Materials and Methods}

\section{Ethical Approval}

This prospective study was conducted in accordance with the amended Declaration of Helsinki and with the approval of our Institutional Review Board (Keio University School of Medicine Ethics Committee, No. 20160384). Written informed consent was obtained from all participants (UMIN Clinical Trials Registry [UMIN-CTR]: UMIN000026586).

\section{Study Population}

From April 2018 to October 2018, 32 asymptomatic volunteers from a volunteer recruitment company, consisting of 16 men and 16 women ( 4 men and 4 women, from the third, fourth, fifth, and sixth decades of life) were enrolled in this study. To evaluate normal whole-body anatomy, volunteers (1) with a history of smoking, diabetes, hypertension, dyslipidemia, or awareness of dysuria; (2) with any symptoms; (3) who had undergone surgery or were currently undergoing treatment; or (4) who were pregnant or possibly pregnant were excluded. The 32 enrolled subjects had been analyzed for a different purpose in a previous study that evaluated the vena cava, aorta, and pelvic floor, but did not evaluate the lungs [7].

\section{CT Imaging Protocol}

All participants underwent both conventional 320-detectorrow CT (Aquilion ONE, Canon Medical Systems, Otawara, Japan) in the supine position (Fig. 1a) and 320-detector-row upright CT (prototype TSX-401R, Canon Medical Systems) [7] in the upright position (Fig. 1b) for the head and body trunk, separately, on the same day. The scan lengths for the head and body trunk were adjusted to be identical between the supine and upright CT scans. Only data for the lung were analyzed in this study. Thirty out of 32 participants underwent conventional CT first, followed by upright $\mathrm{CT}$, and two participants underwent upright CT first, followed by conventional CT.

For the body trunk, both CT examinations were unenhanced and were performed with automatic exposure control (tube current modulation) using a noise index of 15 for a slice thickness of 

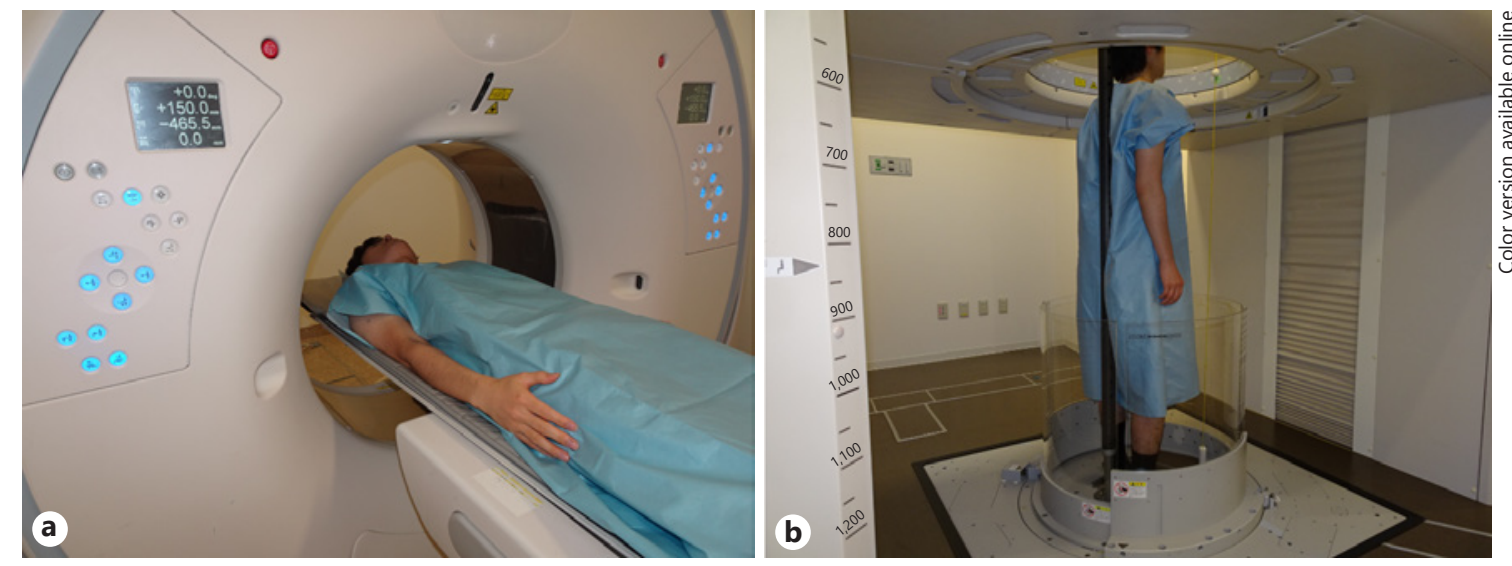

Fig. 1. Conventional CT examination in the supine position (a) and upright CT examination in the standing position (b). a Conventional CT was performed with the subject's arms down during deep inspiration breath-hold. b Upright CT was also performed with the subject's arms down during deep inspiration breath-hold. The upright CT system allows up-and-down movement of a transverse 320-detector-row gantry. For safety during upright CT scanning, an acrylic wall encircling the body was added to prevent falls, with a pinch prevention mechanism and contact interlock control mechanism. Furthermore, to stabilize patients while standing, a back-support pole, 2.3-m long and made of carbon, was included; it was mounted between the floor and the top of the system. The mounting position could be adjusted based on the subject's bodily dimensions or the scan conditions. To support patients who are frail or elderly, a Velcro band can be attached to the pole and loosely wrapped around a patient's body. In this study, the subjects were healthy volunteers; thus, we did not use the Velcro band.
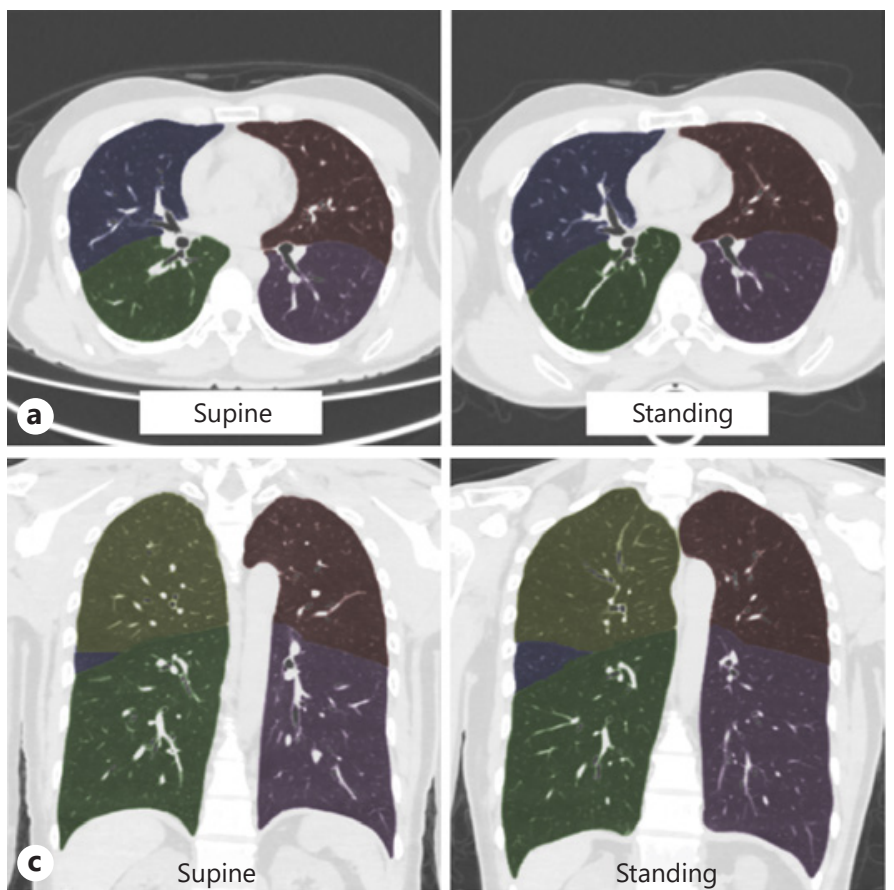

Fig. 2. Representative segmentation and lung/lobe volume measurement in a 44-year-old male: axial (a), sagittal (b), and coronal (c) images, and volume rendering images (d) taken in the supine and standing positions. Yellow is the right upper lobe, blue is the right middle lobe, green is the right lower lobe, pink is the left upper lobe, and purple is the left lower lobe. The volumes of the total (bilateral) lungs in the supine vs. standing positions in this subject
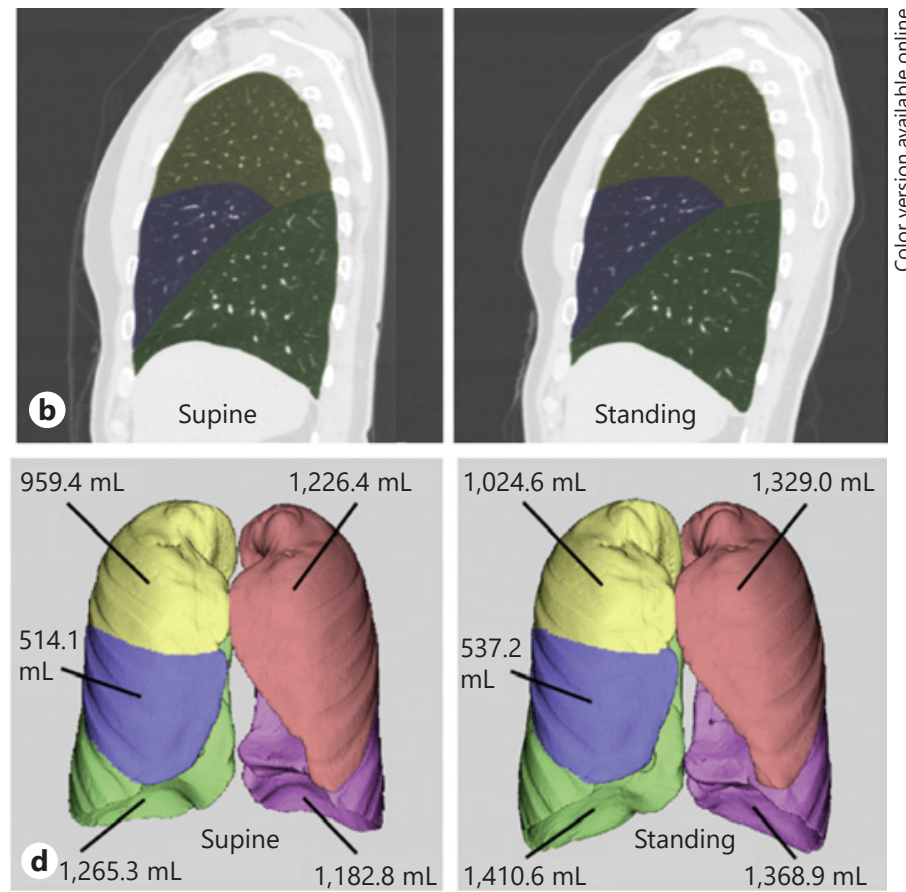

were $5,148.0$ vs. $5,670.2 \mathrm{~mL}$, respectively (10.1\% increase); right lung, $2,738.8$ vs. $2,972.3 \mathrm{~mL}$ ( $8.5 \%$ increase); right upper lobe, 959.4 vs. $1,024.6 \mathrm{~mL}$ (6.8\% increase); right middle lobe, 514.1 vs. 537.2 $\mathrm{mL}$ (4.5\% increase); right lower lobe, $1,265.3$ vs. $1,410.6 \mathrm{~mL}(11.5 \%$ increase); left lung, $2,409.2$ vs. $2,697.9 \mathrm{~mL}$ (12.0\% increase); left upper lobe, $1,226.4$ vs. $1,329.0 \mathrm{~mL}$ (8.4\% increase); and left lower lobe, $1,182.8$ vs. $1,368.9 \mathrm{~mL}$ ( $15.7 \%$ increase $)$. 
$5 \mathrm{~mm}$ (tube current range 10-500 mA). Scans were performed with the subjects' arms down, during deep inspiration breath-hold. This was because the same posture of the arms between supine and standing positions could exclude the changes in the chest anatomical structures caused by the different arm positions. Other scanning parameters were also the same for both supine and standing CT scans: peak tube voltage, $120 \mathrm{kVp}$; rotation speed, $0.5 \mathrm{~s}$; slice collimation, $0.5 \mathrm{~mm} \times 80$; pitch factor, 0.813 . The series of contiguous $0.5-\mathrm{mm}$-thick images were reconstructed with Adaptive Iterative Dose Reduction 3D (Canon Medical Systems) [14].

\section{Pulmonary Function Test}

All participants underwent PFTs on the same day as both conventional and upright CT examinations. The PFT was performed in a stable condition and with the subject in a sitting position, using a spirometer (Chestac-8900, Chest M.I., Tokyo, Japan) in accordance with ATS/European Respiratory Society recommendations [15]. The TLC and residual volume were measured by the multibreath helium dilution method.

\section{Lung and Lobe Volume Measurements on CT}

The first lung and lobe volume measurements on CT for all 32 volunteers in each position were performed by a chest radiologist with 12 years of experience, using a commercially available workstation (Synapse Vincent, Fuji Film Co., Ltd., Tokyo, Japan). This workstation incorporated a lobar computer-aided diagnosis (CAD) system that was previously demonstrated to measure lobar volumes precisely [16]. This system automatically extracted right and left lungs, recognized lobar bronchi, and determined the locations of fissures (Fig. 2). The chest radiologist verified the results of segmentation by $\mathrm{CAD}$ and made manual corrections by delineating fissures when the CAD system failed to identify fissures properly, as described in a previous study [17]. A second measurement of the first 16 volunteers was performed by the same radiologist to assess intraobserver agreement, 1 month after the first assessment. To assess interobserver agreement, lung volume measurements of the first 16 volunteers were performed by a different general radiologist with 5 years of experience. All measurements were performed in a blinded and randomized manner. During all measurements, the radiologists were also blinded to participants' characteristics and the results of the PFT. The ratios of the volume of the total (bilateral) lung, each lung, and each lobe in the standing position to those in the supine position were then calculated. The proportional volumes of each lung and each lobe relative to the total lung volume were also calculated.

\section{Statistical Analysis}

Data are presented as mean \pm SD. The sample-size calculation was based on postural changes in lung volume. A previous study that evaluated the TLC in a volunteer cohort with PFT showed that the difference in TLC between supine and standing positions was 550 $\mathrm{mL}$ (SD $650 \mathrm{~mL}$ ) [8]. To detect clinically significant differences in the CT-based volume of each lung and each lobe between the 2 positions, with a significance level of $5 \%$ (two-tailed) and $90 \%$ power, 30 volunteers were needed for each position group. Paired $t$ tests were used to analyze differences in the volumes of the total lungs, right lung, left lung, and each lobe between supine and standing positions, the differences in ratios of volumes in the standing position to those in the supine position among lobes, and the differences in the proportional volumes of each lung and each lobe relative to the total lung volume between supine and standing positions. Bonfer-
Table 1. Characteristics of the study population ( $n=32$ volunteers)

\begin{tabular}{ll}
\hline Demographic variables & Value \\
\cline { 2 - 3 } & $\begin{array}{l}\text { average } \pm \text { SD range } \\
\text { or } n(\%)\end{array}$ \\
\hline
\end{tabular}

\begin{tabular}{lcc}
$\begin{array}{l}\text { Total number of participants, } n \\
\text { Sex }\end{array}$ & 32 & \\
$\quad$ Female & $16(50)$ & \\
$\quad$ Male & $16(50)$ & \\
Age, years & $48.4 \pm 11.5$ & $30-68$ \\
Height, cm & $163.3 \pm 7.7$ & $147.7-177.0$ \\
Weight, kg & $60.3 \pm 10.8$ & $41.6-88.0$ \\
Body mass index & $22.5 \pm 3.0$ & $16.7-30.6$ \\
Pulmonary function test & & \\
VC, L & $3.71 \pm 0.83$ & $2.51-5.41$ \\
FVC, L & $3.64 \pm 0.83$ & $2.49-5.39$ \\
FVC, \% predicted & $102.9 \pm 11.0$ & $82.9-124.8$ \\
$\quad$ FEV, L & $2.90 \pm 0.66$ & $1.81-4.20$ \\
FEV, \% predicted & $98.7 \pm 12.8$ & $70.6-117.2$ \\
FEV 1 /FVC, \% & $80.1 \pm 6.2$ & $60.1-91.4$ \\
Tidal volume, L & $0.63 \pm 0.22$ & $0.40-1.53$ \\
Residual volume, L & $1.54 \pm 0.36$ & $0.82-2.25$ \\
Functional residual capacity, L & $2.98 \pm 0.73$ & $2.08-4.68$ \\
Total lung capacity, L & $5.25 \pm 1.08$ & $3.45-7.43$ \\
\hline
\end{tabular}

$\mathrm{SD}$, standard deviation; $\mathrm{FEV}_{1}$, forced expiratory volume in $1 \mathrm{~s}$; FVC, forced vital capacity; VC, vital capacity.

roni correction was used for multiple comparisons. Mann-Whitney $\mathrm{U}$ tests were used to analyze the differences in the lung/lobe volumes between conventional CT first group and upright CT first group, and the differences in ratios of volumes in the standing position to those in the supine position between conventional CT first group and upright CT first group. The interobserver and intraobserver agreements were evaluated by measuring the intraclass correlation coefficients (ICCs). The correlation between the total lung volume on CT in each position and TLC on PFT was also evaluated by measuring the ICCs. The significance level for all tests was 5\% (twosided). All data were analyzed using a commercially available software program (JMP version 12; SAS Institute Inc., Cary, NC, USA).

\section{Results}

\section{Participants' Characteristics}

Table 1 shows the clinical characteristics of all the participants $(n=32)$.

\section{Lung and Lobe Volumes on CT in the Supine and Standing Positions}

The average volumes of total lungs (10.9\% increase), right lung (10.3\% increase), right upper lobe $(8.6 \%$ increase), right lower lobe (14.6\% increase), left lung $(11.6 \%$ increase), left upper lobe (7.1\% increase), and left lower 
Table 2. Lung and lobe volumes on CT ( $n=32$ volunteers) in the arms-down supine and standing positions

\begin{tabular}{|c|c|c|c|c|}
\hline & \multicolumn{2}{|c|}{ Lung and lobe volumes on $\mathrm{CT}, \mathrm{mL}$} & \multirow{2}{*}{$\begin{array}{l}p \text { value } \\
\text { supine vs. standing }\end{array}$} & \multirow{2}{*}{$\begin{array}{l}\text { Ratio of volume in } \\
\text { standing position to that } \\
\text { in supine position }\end{array}$} \\
\hline & supine & standing & & \\
\hline Right lung & $\begin{array}{l}2,342.6 \pm 503.5 \\
(1,341.2-3,176.8)\end{array}$ & $\begin{array}{l}2,585.0 \pm 602.6 \\
(1,500.8-3,792.3)\end{array}$ & $<0.0001$ & $\begin{array}{l}1.104 \pm 0.093 \\
(0.901-1.281)\end{array}$ \\
\hline Right upper lobe & $\begin{array}{l}782.0 \pm 200.2 \\
(467.5-1,239.9)\end{array}$ & $\begin{array}{l}849.2 \pm 220.8 \\
(563.5-1,435.5)\end{array}$ & $<0.0001$ & $\begin{array}{l}1.088 \pm 0.072^{\mathrm{a}} \\
(0.944-1.224)\end{array}$ \\
\hline Right middle lobe & $\begin{array}{l}403.5 \pm 109.2 \\
(190.5-635.4)\end{array}$ & $\begin{array}{l}410.4 \pm 113.6 \\
(192.9-645.6)\end{array}$ & 0.1631 & $\begin{array}{l}1.017 \pm 0.067^{\mathrm{a}} \\
(0.850-1.193)\end{array}$ \\
\hline Right lower lobe & $\begin{array}{l}1,157.1 \pm 268.3 \\
(591.8-1,602.2)\end{array}$ & $\begin{array}{l}1,325.5 \pm 336.4 \\
(669.0-2,103.0)\end{array}$ & $<0.0001$ & $\begin{array}{l}1.148 \pm 0.129^{a} \\
(0.892-1.405)\end{array}$ \\
\hline Left upper lobe & $\begin{array}{l}1,012.9 \pm 253.4 \\
(581.1-1,446.2)\end{array}$ & $\begin{array}{l}1,084.5 \pm 269.4 \\
(666.6-1,562.4)\end{array}$ & $<0.0001$ & $\begin{array}{l}1.075 \pm 0.078^{b} \\
(0.909-1.272)\end{array}$ \\
\hline Left lower lobe & $\begin{array}{l}1,023.2 \pm 265.3 \\
(530.0-1,475.3)\end{array}$ & $\begin{array}{l}1,187.0 \pm 322.4 \\
(667.7-1,775.5)\end{array}$ & $<0.0001$ & $\begin{array}{l}1.167 \pm 0.141^{\mathrm{b}} \\
(0.896-1.471)\end{array}$ \\
\hline
\end{tabular}

Values are presented as average \pm standard deviation (range). ${ }^{\text {a }}$ The ratio of right lower lobe volume in the standing position to that in the supine position was significantly greater than that of the right upper lobe volume $(p<0.0001)$ and that of the right middle lobe volume $(p<0.0001)$; the ratio of the right upper lobe volume in the standing position to that in the supine position was significantly greater than that of the right middle lobe volume $(p<0.0001)$. A value of $p<0.0167$ was considered to be statistically significant after Bonferroni correction for multiple comparisons. ${ }^{b}$ The ratio of the left lower lobe volume in the standing position to that in the supine position was significantly greater than that of the left upper lobe volume $(p<0.0001)$.

lobe $(16.0 \%$ increase) in the standing position were significantly greater than those in the supine position (all $p<0.0001)$. There was no significant difference in right middle lobe volume (1.7\% difference) between the two positions $(p=0.16)$ (Table 2$)$.

The ratio of the right lower lobe volume in the standing position to that in the supine position $(1.148 \pm 0.129)$ was significantly greater than that of the right upper lobe volume $(1.088 \pm 0.072 ; p<0.0001)$ and that of the right middle lobe volume $(1.017 \pm 0.067 ; p<0.0001)$. The ratio of the right upper lobe volume in the standing position to that in the supine position was also significantly greater than that of the right middle lobe volume $(p<0.0001 ; \mathrm{Ta}-$ ble 2). The ratio of the left lower lobe volume in the standing position to that in the supine position $(1.167 \pm 0.141)$ was significantly greater than that of the left upper lobe volume $(1.075 \pm 0.078 ; p<0.0001)$. Subanalyses showed that there were no significant differences in all lung/lobe volumes in the supine or standing position between conventional CT first group and upright CT first group (all $p>0.45)$ and that there were no significant differences in all ratios of lung/lobe volumes in the standing position to those in the supine position between conventional CT first group and upright CT first group (all $p>0.15$ ).

\section{Proportional Volumes of Each Lung and Each Lobe relative to the Total Lung Volume in Supine and Standing Positions}

The proportional volumes of the bilateral upper lobes and right middle lobe relative to the total lung volume were significantly lower in the standing position than in the supine position, whereas the proportional volumes of the bilateral lower lobes were significantly greater in the standing position than in the supine position (all $p<$ 0.002; Table 3).

\section{Agreement between Total Lung Volumes on CT in}

Supine/Standing Positions and TLC on PFT

The ICC for agreement between the total lung volume on CT in the standing position and TLC on PFT was nu- 
Table 3. Proportional volumes of each lung and each lobe relative to the total lung volume on CT in the supine and standing positions ( $n=32$ volunteers)

\begin{tabular}{clll}
\hline & $\begin{array}{l}\text { Proportional volumes of each } \\
\text { lung and each lobe relative to } \\
\text { the total lung volume, } \%\end{array}$ & $\begin{array}{l}p \text { value } \\
\text { supine } \\
\text { vs. } \\
\text { standing }\end{array}$ \\
\cline { 2 - 3 } supine & standing & - \\
\hline Total (bilateral) lung & $\begin{array}{l}100.0 \pm 0.0 \\
(100.0-100.0)\end{array}$ & $\begin{array}{l}100.0 \pm 0.0 \\
(100.0-100.0)\end{array}$ & 0.0467 \\
\hline Right lung & $\begin{array}{l}53.6 \pm 1.5 \\
(50.6-57.4)\end{array}$ & $\begin{array}{l}53.3 \pm 1.3 \\
(50.5-56.3)\end{array}$ & 0.0011 \\
\hline Right upper lobe & $\begin{array}{l}17.9 \pm 2.4 \\
(11.8-22.7)\end{array}$ & $\begin{array}{l}17.6 \pm 2.3 \\
(12.0-21.9)\end{array}$ & $<0.0001$ \\
\hline Right middle lobe & $\begin{array}{l}9.3 \pm 1.6 \\
(6.6-13.8)\end{array}$ & $\begin{array}{l}8.5 \pm 1.4 \\
(6.1-11.6)\end{array}$ & 0.0003 \\
\hline Right lower lobe & $\begin{array}{l}26.5 \pm 2.6 \\
(22.3-34.0)\end{array}$ & $\begin{array}{l}27.2 \pm 2.2 \\
(22.4-33.7)\end{array}$ & 0.0470 \\
\hline Left lung & $\begin{array}{l}46.4 \pm 1.5 \\
(42.6-49.4)\end{array}$ & $\begin{array}{l}46.7 \pm 1.3 \\
(43.7-49.5)\end{array}$ & $<0.0001$ \\
\hline Left upper lobe & $\begin{array}{l}23.1 \pm 2.1 \\
(18.1-26.6)\end{array}$ & $\begin{array}{l}22.4 \pm 2.0 \\
(17.1-25.8)\end{array}$ & \\
\hline Left lower lobe & $\begin{array}{l}23.2 \pm 2.4 \\
(19.4-28.7)\end{array}$ & $\begin{array}{l}24.3 \pm 2.4 \\
(20.7-29.6)\end{array}$ & $<0.0001$ \\
\hline
\end{tabular}

Values are presented as average \pm standard deviation (range).

merically greater than that for agreement between the total lung volume on CT in the supine position and TLC on PFT (0.938 [95\% CI 0.877-0.969] vs. 0.891 [95\% CI $0.789-0.945])$.

\section{Interobserver and Intraobserver Agreements}

The interobserver and intraobserver agreements (ICCs) for measuring lung and lobe volumes on CT in the supine and standing positions were substantial for all measurements (0.978-1.000; Table 4).

\section{Discussion}

Our prospective study demonstrated that the volumes of the bilateral upper lobes, bilateral lower lobes, bilateral lungs, and total lungs were significantly greater in the standing position than in the supine position, with lower lobes showing larger changes, while there was no significant difference in the volume of the right middle lobe between the supine and standing positions. Consequently, the proportional volumes of the bilateral upper lobes and right middle lobe relative to the total lung vol- ume were significantly lower in the standing position than in the supine position, whereas the proportional volumes of bilateral lower lobes were significantly greater in the standing position than in the supine position. Our findings are important because they could have an impact on preoperative CT volumetry of the lung in patients with lung cancer. Upright CT provides images of daily-life postures (both standing and sitting positions) and is potentially useful for a more accurate prediction of postoperative residual pulmonary function, before lung resection, as compared with conventional CT (supine position).

We found that the volume increase in the lower lobe, in the standing position, was greater than that in the upper lobe or middle lobe. The lower lungs undergo greater volume changes during breathing than the upper lungs, due to the effects of gravity on lung recoil [18-20]. Thus, a possible reason for the greater increase in the lower lobes could be that the diaphragm settles lower in the standing position than in the supine position, which allows for greater expansion of the lower lobes. Several reports showed that loss of the diaphragmatic mobility was present in chronic obstructive pulmonary disease (COPD) patients [21-23], and pulmonary rehabilitation improved diaphragm movement $[21,24]$, which was assessed in standing or semi-recumbent position. The volumes of lung lower lobes are influenced by diaphragm movement. Thus, the volumes of lung lower lobes in the standing position or change of the volumes between supine and standing positions may be used as a new clinical indicator to assess disease severity or therapeutic effect in COPD patients. Our results also showed that there was no significant difference in volume of the right middle lobe between the two positions. Although the reason for this is not immediately clear, we speculate that the right middle lobe has a relatively small volume and is located in a relatively anterior portion of the right lung in the standing position and could be compressed by an enlarged lower lobe, with a volume approximately 3 times as large as that of the middle lobe, in the standing position.

Our results demonstrated that the proportional volumes of each lobe relative to the total lung volume were different in all lobes between supine and standing positions. Although further studies in larger patient populations are needed, a corrective model based on our results of these proportional volumes and the ratio of total lung volume in standing position to that in supine position may allow for the prediction of the lobe or lung volume in the standing position from the lobe or lung volume in 
Table 4. Interobserver and intraobserver agreements (ICCs) for measuring lung and lobe volumes on CT in the supine and standing positions ( $n=32$ volunteers)

\begin{tabular}{|c|c|c|c|c|}
\hline & \multicolumn{2}{|c|}{$\begin{array}{l}\text { Interobserver agreements for measuring lung } \\
\text { and lobe volumes ( } 95 \% \text { confidence interval) }\end{array}$} & \multicolumn{2}{|c|}{$\begin{array}{l}\text { Intraobserver agreements for measuring lung } \\
\text { and lobe volumes ( } 95 \% \text { confidence interval) }\end{array}$} \\
\hline & supine & standing & supine & standing \\
\hline Total (bilateral) lung & $1.000(1.000-1.000)$ & $1.000(1.000-1.000)$ & $1.000(1.000-1.000)$ & $1.000(1.000-1.000)$ \\
\hline Right lung & $1.000(1.000-1.000)$ & $1.000(1.000-1.000)$ & $1.000(1.000-1.000)$ & $1.000(1.000-1.000)$ \\
\hline Right upper lobe & $0.999(0.997-1.000)$ & $0.994(0.983-0.998)$ & $1.000(0.999-1.000)$ & $0.994(0.983-0.998)$ \\
\hline Left lung & $1.000(1.000-1.000)$ & $1.000(1.000-1.000)$ & $1.000(1.000-1.000)$ & $1.000(1.000-1.000)$ \\
\hline Left upper lobe & $0.999(0.997-1.000)$ & $0.999(0.997-1.000)$ & $0.999(0.997-1.000)$ & $0.999(0.998-1.000)$ \\
\hline Left lower lobe & $0.999(0.998-1.000)$ & $0.999(0.998-1.000)$ & $0.999(0.998-1.000)$ & $1.000(0.999-1.000)$ \\
\hline
\end{tabular}

the supine position acquired by conventional CT or the TLC on PFT.

Our study also demonstrated that the total lung volume determined using upright $\mathrm{CT}$ in the standing position was more similar to TLC on PFT than that determined in the supine position (ICC, 0.938 vs. 0.891 ). This may be because the PFT was performed in the upright (sitting) position, and the direction of the thorax in the PFT was more similar to that in upright CT than in conventional CT (supine position).

Our study had several limitations. First, we included only 32 normal subjects at a single institution to avoid unnecessary radiation exposure to the volunteer cohort. As mentioned above, further studies in larger patient populations are required to confirm the preliminary findings of this study. Nevertheless, upright CT has only been introduced at our hospital at present. Second, although the observers independently evaluated the images in a blinded and randomized manner, they could recognize the positions of the subjects to some extent because of the presence or absence of a CT table. However, the measurement of lung and lobe volumes was semiautomated, using a commercially available workstation, and the observer bias was negligible. Furthermore, the interobserver and intraobserver agreements (ICCs) were quite high (>0.97) in this study. Third, the supine CT was performed with the subject's arms down to maintain the same posture as that during the standing position, although a conventional chest CT in the supine position is usually performed with the subject's arms raised.

\section{Conclusions}

The CT-based volumes of bilateral upper lobes, bilateral lower lobes, bilateral lungs, and total lungs were significantly greater in the standing position than in the supine position, with lower lobes showing larger changes, while the right middle lobe showed no significant change between the two positions. The total lung volume on upright CT in the standing position was more similar to TLC on PFT than that on conventional CT in the supine position.

\section{Acknowledgement}

The authors would like to acknowledge all of the volunteers for their willingness to participate in the study. The authors thank Naomi Tamaki and Kyoko Komatsu for their contributions to the manuscript preparation.

\section{Statement of Ethics}

This study was conducted in accordance with the amended Declaration of Helsinki and with the approval of our Institutional Review Board (Keio University School of Medicine Ethics Committee, No. 20160384). Written informed consent was obtained from all participants.

\section{Conflict of Interest Statement}

M.J. has received a grant from Canon Medical Systems. However, Canon Medical Systems was not involved in the design and conduct of the study, in the collection, analysis, and interpretation of the data, or in the preparation, review, and approval of the manuscript. The remaining authors have no conflicts of interest to declare (Y. Yamada, M.Y., Y. Yokoyama, A.T., S.M., Y.N., K.N., T.N., M.M., K.F., and S.C.). 


\section{Funding Sources}

This study was supported by Japan Society for the Promotion of Science (JSPS) KAKENHI (grant number 17H04266, 20K08056, and 17K16482), Uehara Memorial Foundation, and Canon Medical Systems (Otawara, Japan).

\section{Author Contributions}

Y. Yamada, M.Y., Y. Yokoyama, and M.J. were responsible for the conception or design of the work; Y. Yamada, M.Y., Y. Yokoyama, A.T., S.M., Y.N., K.N., and T.N. were responsible for the acquisition of data; Y. Yamada, M.Y., Y. Yokoyama, and S.C. analyzed and interpreted the data; Y. Yamada, M.Y., Y. Yokoyama, A.T., S.M., Y.N., K.N., T.N., M.M., K.F., S.C., and M.J. drafted or revised the paper for important intellectual content and provided final approval of the data; Y. Yamada and M.J. are accountable for all aspects of the work.

\section{References}

1 Segal NA, Bergin J, Kern A, Findlay C, Anderson DD. Test-retest reliability of tibiofemoral joint space width measurements made using a low-dose standing CT scanner. Skeletal Radiol. 2017 Feb;46(2):217-22.

2 Demehri S, Muhit A, Zbijewski W, Stayman JW, Yorkston J, Packard N, et al. Assessment of image quality in soft tissue and bone visualization tasks for a dedicated extremity conebeam CT system. Eur Radiol. 2015 Jun;25(6): 1742-51.

3 Kiljunen T, Kaasalainen T, Suomalainen A, Kortesniemi M. Dental cone beam CT: a review. Phys Med. 2015 Dec;31(8):844-60.

4 Adibi S, Zhang W, Servos T, O’Neill PN. Cone beam computed tomography in dentistry: what dental educators and learners should know. J Dent Educ. 2012 Nov;76(11):143742.

5 de Smet M, Schuring D, Nijsten S, Verhaegen F. Accuracy of dose calculations on $\mathrm{kV}$ cone beam CT images of lung cancer patients. Med Phys. 2016 Nov;43(11):5934-41.

6 Clarke E, Curtis J, Brada M. Incidence and evolution of imaging changes on cone-beam CT during and after radical radiotherapy for non-small cell lung cancer. Radiother Oncol. 2019 Mar;132:121-6.

7 Jinzaki M, Yamada Y, Nagura T, Nakahara T, Yokoyama Y, Narita K, et al. Development of upright computed tomography with area detector for whole-body scans: phantom study, efficacy on workflow, effect of gravity on human body, and potential clinical impact. Invest Radiol. 2020 Feb;55(2):73-83.

8 Blair E, Hickam JB. The effect of change in body position on lung volume and intrapulmonary gas mixing in normal subjects. J Clin Invest. 1955 Mar;34(3):383-9.

9 Talaminos Barroso A, Márquez Martín E, Roa Romero LM, Ortega Ruiz F. Factors affecting lung function: a review of the literature. Arch Bronconeumol. 2018 Jun;54(6):327-32.
10 Kobayashi K, Saeki Y, Kitazawa S, Kobayashi N, Kikuchi S, Goto Y, et al. Three-dimensional computed tomographic volumetry precisely predicts the postoperative pulmonary function. Surg Today. 2017 Nov;47(11):1303-11.

11 Yoshimoto K, Nomori H, Mori T, Kobayashi $\mathrm{H}$, Ohba Y, Shibata H, et al. Prediction of pulmonary function after lung lobectomy by subsegments counting, computed tomography, single photon emission computed tomography and computed tomography: a comparative study. Eur J Cardiothorac Surg. 2009 Mar; 35(3):408-13.

12 Wu MT, Chang JM, Chiang AA, Lu JY, Hsu $\mathrm{HK}, \mathrm{Hsu} \mathrm{WH}$, et al. Use of quantitative CT to predict postoperative lung function in patients with lung cancer. Radiology. 1994 Apr; 191(1):257-62.

13 Tenda ED, Ridge CA, Shen M, Yang GZ, Shah PL. Role of quantitative computed tomographic scan analysis in lung volume reduction for emphysema. Respiration. 2019;98(1): 86-94.

14 Yamada Y, Jinzaki M, Hosokawa T, Tanami Y, Sugiura H, Abe T, et al. Dose reduction in chest CT: comparison of the adaptive iterative dose reduction $3 \mathrm{D}$, adaptive iterative dose reduction, and filtered back projection reconstruction techniques. Eur J Radiol. 2012 Dec; 81(12):4185-95.

15 Miller MR, Hankinson J, Brusasco V, Burgos F, Casaburi R, Coates A, et al.; ATS/ERS Task Force. Standardisation of spirometry. Eur Respir J. 2005 Aug;26(2):319-38.

16 Iwano S, Kitano M, Matsuo K, Kawakami K, Koike W, Kishimoto M, et al. Pulmonary lobar volumetry using novel volumetric computer-aided diagnosis and computed tomography. Interact Cardiovasc Thorac Surg. 2013 Jul;17(1):59-65.

17 Kitano M, Iwano S, Hashimoto N, Matsuo K, Hasegawa Y, Naganawa S. Lobar analysis of collapsibility indices to assess functional lung volumes in COPD patients. Int J Chron Obstruct Pulmon Dis. 2014 Dec;9:1347-56.
18 Ratnovsky A, Elad D, Halpern P. Mechanics of respiratory muscles. Respir Physiol Neurobiol. 2008 Nov; 163(1-3):82-9.

19 Tanaka R, Sanada S, Okazaki N, Kobayashi T, Suzuki M, Matsui T, et al. Detectability of regional lung ventilation with flat-panel detector-based dynamic radiography. J Digit Imaging. 2008 Mar;21(1):109-20.

20 Yamada $\mathrm{Y}$, Ueyama M, Abe T, Araki T, Abe $\mathrm{T}$, Nishino $\mathrm{M}$, et al. Difference in the craniocaudal gradient of the maximum pixel value change rate between chronic obstructive pulmonary disease patients and normal subjects using sub-mGy dynamic chest radiography with a flat panel detector system. Eur J Radiol. 2017 Jul;92:37-44.

21 Corbellini C, Boussuges A, Villafañe JH, Zocchi L. Diaphragmatic mobility loss in subjects with moderate to very severe COPD may improve after in-patient pulmonary rehabilitation. Respir Care. 2018 Oct;63(10):1271-80.

22 Hida T, Yamada Y, Ueyama M, Araki T, Nishino M, Kurosaki A, et al. Decreased and slower diaphragmatic motion during forced breathing in severe COPD patients: time-resolved quantitative analysis using dynamic chest radiography with a flat panel detector system. Eur J Radiol. 2019 Mar;112:28-36.

23 Yamada Y, Ueyama M, Abe T, Araki T, Abe $\mathrm{T}$, Nishino $\mathrm{M}$, et al. Difference in diaphragmatic motion during tidal breathing in a standing position between COPD patients and normal subjects: time-resolved quantitative evaluation using dynamic chest radiography with flat panel detector system ("dynamic X-ray phrenicography”). Eur J Radiol. 2017 Feb;87:76-82.

24 Chun EM, Han SJ, Modi HN. Analysis of diaphragmatic movement before and after pulmonary rehabilitation using fluoroscopy imaging in patients with COPD. Int $\mathrm{J}$ Chron $\mathrm{Ob}-$ struct Pulmon Dis. 2015 Jan;10:193-9. 